\title{
"Mal del ciprés": búsqueda de la causa más probable de daño mediante un análisis deductivo y comparativo
}

\author{
"Cypress Mortality": search the more probably damage cause by means of \\ deductive and comparative analysis
}

\author{
Verónica Andrea El Mujtara* y Ernesto Andenmatten ${ }^{b}$ \\ *Autor de correspondencia: ${ }^{a}$ Universidad Nacional de La Plata, Facultad de Ciencias Exactas, Instituto de Bioquímica y \\ Biología Molecular (IBBM), Calle 49 y 115 s/n (1900), La Plata, Buenos Aires, Argentina, Tel./Fax: 54-221 4259223, \\ verel@biol.unlp.edu.ar. \\ b Instituto Nacional de Tecnología Agropecuaria (INTA)-EEA, Bariloche, Argentina.
}

\begin{abstract}
SUMMARY
The "cypress mortality" ("mal del ciprés") is a disease of unknown causes, which affects cordillera cypress (Austrocedrus chilensis) in Argentina. In this work a basic guide to diagnosing plant diseases and to compare the cypress mortality with other forest diseases is shown for its application, in order to identify the most probable cause of damage. The systematic management of the information permitted to establish that the dates are not sufficient to discriminate between different damage agents. The association between the cypress mortality and others forest diseases allowed us to verify that there are several difficulties to diagnose and manage this pathologies. We detected several key points of the disease that should be considered in future investigations: absence of specific symptoms, absence of signs, subjective assignment of the degree of defoliation and the associated sanitary state, absence of an analysis model for disturbance.
\end{abstract}

Key words: Austrocedrus chilensis, guide to diagnosing, deductive steps, propagation study, analysis models.

\section{RESUMEN}

El "mal del ciprés" es un disturbio de origen desconocido que afecta a ciprés de la cordillera (Austrocedrus chilensis) en Argentina. En este trabajo se plantea la aplicación de una guía básica para el diagnóstico de enfermedades de plantas con el fin de identificar la causa más probable de daño. Por otra parte, se compara el "mal del ciprés" con algunas enfermedades de especies forestales. El manejo sistemático de la información, logrado al aplicar la guía de diagnóstico, permitió establecer que los datos existentes son insuficientes para discriminar entre los diferentes grupos de agentes de daño. La comparación entre el "mal del ciprés" y diferentes enfermedades de especies forestales permitió comprobar que son muchas las dificultades que encierra el diagnóstico y el manejo de tales patologías. De la implementación de ambos enfoques surgieron algunos puntos claves de la afección que deberían ser considerados en investigaciones futuras: falta de especificidad de síntomas, falta de estudios de propagación, ausencia de signos de la afección, subjetividad en la asignación del grado de defoliación y el estado sanitario asociado, falta de un modelo de análisis para el disturbio.

Palabras clave: Austrocedrus chilensis, guía de diagnóstico, pasos deductivos, estudios de propagación, modelo de análisis.

\section{INTRODUCCIÓN}

Austrocedrus chilensis (D. Don) Pic. Ser et Bizzarri (ciprés de la cordillera) se distribuye en una franja de no más de $50 \mathrm{~km}$ de ancho, formada por una serie de parches de bosque de tamaño variable. En Chile se encuentra entre los límites $32^{\circ} 39^{\prime} \mathrm{S}$ y $44^{\circ} \mathrm{S}$, mientras que en Argentina lo hace entre los $39^{\circ} 30^{\prime} \mathrm{S}$ y $43^{\circ} 44^{\prime} \mathrm{S}$ (Gallo et al. 2004). La especie está adaptada a un amplio rango de condiciones ambientales y sus bosques se localizan en un marcado gradiente de precipitación. Los bosques de ciprés de la cordillera forman el denominado "Cinturón de Frontera" entre los bosques húmedos de Nothofagus spp. al oeste y la estepa patagónica al este (Dezzotti y Sancholuz 1991).

Un disturbio de origen desconocido, denominado "mal del ciprés", afecta a esta especie en Argentina. De acuerdo a los datos históricos, los primeros registros se 
localizan en Puerto Piedras Blancas (Isla Victoria, Provincia de Neuquén) en el año 1948 (Varsavsky et al. 1975) y en cipresales adyacentes al Vivero Forestal General San Martín (Lote 12, Sección Golondrinas, Colonia Mixta; Epuyén al NO de la Provincia de Chubut) en el año 1953 (Hranilovich 1988). En la actualidad, la afección está presente en casi toda el área de distribución del ciprés de la cordillera y el número de árboles afectados es cada vez mayor (Havrylenko et al. 1989).

Havrylenko et al. (1989) señalan una sintomatología con características identificables y constantes en todos los bosques afectados, sin signos visibles y con un patrón de síntomas sin relación aparente con afecciones conocidas. Concluyen que la afección responde a una misma causa independientemente de los bosques estudiados, con inicio aparente en la zona radical, existiendo factores de sitio que favorecen el desarrollo y sin incidencia preferencial según edad de los árboles. Indican, además, que los hongos xilófagos asociados a las pudriciones radiculares no son responsables principales de la mortalidad del ciprés, sino que atacan un sistema ya debilitado por otro agente.

Rosso et al. (1989) indican la existencia de segregación mutua entre los individuos sanos y afectados, postulando que la mortalidad se dispondría en forma agregada debido a un proceso de contagio. En un trabajo posterior señalan que la mortalidad del ciprés de la cordillera se presenta en forma de manchones cuando la incidencia de la afección es baja, y que las condiciones del ambiente en cada parcela influyen en forma directa sobre el grado de agregación cambiando el patrón espacial (Rosso et al. 1994).

El estudio desarrollado por Baccalá et al. (1998) establece la existencia de un patrón ecológico en el disturbio según el cual los bosques más propensos a desarrollar síntomas serían los que se ubican en sitios con mayores precipitaciones y peor drenaje. Calí (1996) indica que la manifestación externa de la afección se correlaciona con una caída del crecimiento radial y que el inicio de dicha caída se da, por lo general, varios años antes que la sintomatología externa en la copa. También indica afección asociada a sitios húmedos y pequeños cursos de agua y con menor grado de correlación a la actividad humana y la pendiente del terreno.

Rajchenberg y Cwielong (1993) sostienen que las pudriciones cúbicas presentes en la albura están fuertemente relacionadas con el "mal del ciprés". Indican a las especies Coniophora arida y Postia dissecta como causantes de estas pudriciones y postulan que se comportarían como parásitos oportunistas. Establecen, además, que las condiciones del sitio jugarían un rol principal en el establecimiento de la afección. Y concluyen que suelos con un horizonte "A" poco profundo, seguido de un horizonte arcilloso impermeable al paso del agua, y terrenos con poca pendiente, estarían asociados con una mayor incidencia del disturbio.
La Manna et al. (2001) mencionan la presencia de lesiones necróticas en raíces de árboles afectados, probablemente producidas por hongos de los géneros Phytophthora y Pythium. Y establecen que la naturaleza del suelo condiciona la forma más o menos agregada en que se presenta la afección. Existe también un importante trabajo de aislamiento y caracterización de especies del género Phytophthora en bosques de ciprés de la cordillera y cursos de agua asociados (Greslebin et al. 2005).

Hasta la fecha se ha logrado:

caracterizar la sintomatología: marchitamiento y amarillamiento progresivo de las hojas, defoliación del árbol, disminución del crecimiento, pudrición intensa de las raíces principales, pudrición en tronco (proveniente de las raíces y con escasa extensión) y muerte en pie.

O comprobar que son afectados individuos de distintas edades, tamaños y posiciones sociológicas.

establecer que la afección aparece como manchones más o menos extendidos (aunque la presentación dentro de cada manchón es más difícil de definir y depende en gran parte de las características del sitio y de la incidencia de la misma).

O establecer la relación entre las condiciones del sitio y la aparición del disturbio.

demostrar la utilidad de las técnicas dendrocronológicas para determinar el inicio de alteraciones en la planta en forma previa a la aparición de síntomas externos.

A pesar de toda la investigación desarrollada, la etiología del disturbio es aún desconocida. La probabilidad de un diagnóstico correcto basado sólo en unos pocos indicios o síntomas es baja, ya que síntomas similares pueden ser producidos por diferentes agentes de daño en una misma planta. El uso exclusivo de la sintomatología como elemento de diagnóstico es inadecuado, ya que la presencia de signos y el patrón de presentación y propagación del daño deben también ser utilizados para discernir entre diferentes agentes.

En este trabajo se aplicó una "guía básica para el diagnóstico de enfermedades de plantas" con el fin de manejar la información existente de un modo sistemático, intentando discriminar entre los probables agentes etiológicos o estableciendo los aspectos que falta investigar para poder lograrlo. De las distintas guías de diagnóstico encontradas, se utilizó la presentada por Green et al. (2003). Esta guía fue seleccionada por la claridad en el desarrollo, por ser aplicable a diversos sistemas y por ajustarse a modelos generales de análisis de enfermedades de plantas, y será seguida sin modificación. Los pasos principales son indicados a continuación (la guía completa puede consultarse en la referencia bibliográfica): definir el problema, búsqueda de patrones, delinear el tiempo de desarrollo del patrón de daño, determinar 
las causas de daño a la planta y sintetizar la información para determinar las posibles causas.

El "mal del ciprés" fue comparado con enfermedades de otras especies forestales en un intento de establecer las dificultades generales que encierra el diagnóstico y el manejo de la investigación de estos disturbios.

\section{APLICACIÓN DE LA GUÍA BÁSICA DE DIAGNÓSTICO AL "MAL DEL CIPRÉS"}

Definir el problema. La existencia del problema ha sido ya establecida. La identificación de plantas afectadas puede hacerse a través de síntomas externos (amarillamiento, defoliación) o internos (caída en la tasa de crecimiento). En el trabajo de Havrylenko et al. (1989) se definen los síntomas de la afección, se indica la ausencia de signos visibles y se postula a la zona radical como punto de inicio del daño, con factores de sitio favoreciendo el desarrollo. El inicio en la zona radical y el efecto de las características del sitio han sido también planteados por Rajchenberg y Cwielong (1993), La Manna et al. (2001) y La Manna y Rajchenberg (2004ab). El amarillamiento y la caída de hojas son más intensos que lo esperable para la especie y los patrones de defoliación observados son característicos e identificables (Havrylenko et al. 1989, Rajchenberg y Cwielong 1993). La disminución de la tasa de crecimiento, determinada por dendrocronología (Calí 1996), permite establecer diferencias entre individuos con y sin síntomas aéreos y verificar que la caída del crecimiento radial se inicia varios años antes que la sintomatología externa.

Es importante mencionar que, además de la sintomatología definida como característica para el "mal del ciprés", se observa otro patrón de afección. En este último caso, la coloración de las hojas pasa de clorótica a roja y el árbol muere rápidamente reteniendo aún el follaje (Filip y Rosso 1999). No se sabe si éstas son diferentes manifestaciones de la misma afección o si corresponden a disturbios distintos.

No se ha encontrado bibliografía en la que se relacione el "mal del ciprés" con afecciones simultáneas de otras especies forestales, arbustivas o herbáceas. Este análisis podría ser de mucha utilidad al evaluar el efecto de agentes abióticos en el proceso (sequía, anegamiento, radiación, ozono) y ayudaría a discriminar entre daño producido por agentes bióticos o abióticos.

Búsqueda de patrones de daño. La distinción entre factores de daño bióticos o abióticos se inicia con la caracterización del patrón de afección, aunque no siempre sea esto suficiente. En el "mal del ciprés" se observa un amarillamiento y marchitamiento progresivo, seguido por pérdida de hojas. La defoliación ocurre, en general, de abajo hacia arriba y de adentro hacia afuera de la copa. Havrylenko et al. (1989) establecen que la distribución y el avance de la defoliación responden a seis patrones diferentes: follaje completo, defoliación proximal en ramas inferiores, defoliación total en ramas inferiores, defoliación proximal generalizada, defoliación lateral, defoliación total. Rajchenberg y Cwielong (1993) plantean una tabla de asignación de tipos de vitalidad, según el porcentaje de defoliación (0-10, 10-25, 25-60, 60-99, $100 \%$ ) y las características del árbol (copa, aspecto y coloración de hojas, aspecto de la corteza). Dichos sistemas no son exactamente iguales y la interpretación de los resultados de los trabajos de investigación podría variar al cambiar el sistema de asignación de patrones y tipos de vitalidad.

La investigación desarrollada hasta la fecha indica que las pudriciones de raíz no son responsables principales de la afección. El origen de las lesiones necróticas que se encuentran en las raíces de los árboles está siendo investigado. De la misma forma, la asociación con factores de sitio es difícil de interpretar. Si bien se demuestra que el drenaje pobre del suelo es una característica de gran importancia para el desarrollo de la afección, no se ha podido establecer el fundamento básico de esta asociación. Diferenciar el origen primario o secundario de la defoliación, parece ser un punto de gran importancia.

Delinear el tiempo de desarrollo del patrón de daño. Observar el desarrollo del patrón de daño en el tiempo, es otro elemento clave para distinguir entre factores bióticos y abióticos. Por lo general, los organismos bióticos se multiplican con el tiempo, de forma tal que el daño se propaga en la planta o en grupos de plantas de manera progresiva. Los factores abióticos, generalmente, producen daño en un momento dado y no se propagan en el tiempo. Para el "mal del ciprés" se han realizado algunos estudios de asociación espacial entre individuos sanos y afectados y se ha determinado la forma de presentación de la afección (Rosso et al. 1989, 1994). Sin embargo, no se cuenta con estudios de propagación del disturbio. De acuerdo a los datos históricos, el problema se registró inicialmente en la zona de Isla Victoria (Río Negro), luego en el noroeste de la provincia de Chubut y posteriormente en el resto del área de distribución del ciprés de la cordillera. Estos son datos de registros de aparición del problema y no deben ser considerados como estudios epidemiológicos o de propagación.

Determinar las causas de daño a la planta y sintetizar la información. Los puntos anteriores del modelo deberían permitir la discriminación entre dos grandes grupos de agentes de daño: bióticos y abióticos. Una vez hecha esta distinción se podría avanzar tratando de identificar un agente específico dentro del grupo de agentes de daño seleccionado. En el caso del "mal del ciprés", la información recabada no permite hacer la discriminación entre grupos de agentes. De acuerdo a los datos aportados, los síntomas mejor conocidos del "mal del ciprés" son la caída en la tasa de crecimiento, el amarillamiento y la 
defoliación. La caída en la tasa de crecimiento ha sido determinada por medio de técnicas dendrocronológicas, pero aún falta establecer la causa o las causas que la provocan y evaluar cada una de las hipótesis que han sido planteadas. El amarillamiento y la defoliación son síntomas muy inespecíficos y diversas causas podrían originarlos. El estudio o análisis del patrón de defoliación permitirá una mejor definición del síndrome y posiblemente ayudará a discriminar entre los probables agentes de daño. No se han señalado aún signos de la enfermedad. La investigación mediante técnicas de microscopía óptica y electrónica de diversos tejidos de plantas con y sin síntomas aéreos podría revelar signos que se relacionen con agentes o grupos de agentes específicos.

No es posible, por tanto, definir el tipo de agentes que se asocian con el disturbio. Aún falta información para establecer si el origen de la afección es biótico o abiótico, o si más de un agente (del mismo o de distinto origen) está participando en el desarrollo de la afección.

\section{EL "MAL DEL CIPRÉS" Y OTRAS ENFERMEDADES FORESTALES}

El número de enfermedades que afectan a especies forestales es muy elevado, como lo es también el número de causas o factores que las originan. En este apartado se pretende mencionar y relacionar con el "mal del ciprés" algunas enfermedades forestales que tienen etiología desconocida o que han sido muy difíciles de abordar por las características de su presentación.

Filip y Rosso (1999) comparan el "mal del ciprés" con declinamientos y enfermedades de bosques en América del Norte, discuten algunas hipótesis que han sido planteadas e indican algunos de los estudios que deberían ser conducidos en la investigación. La mortalidad y el declinamiento de Chamaecyparis nootkatensis (D. Don) Spach es una de las primeras enfermedades que ha sido relacionada con el "mal del ciprés". Hennon y Rajchenberg (2000) realizan una interesante comparación entre ambas. La etiología del declinamiento de C. nootkatensis es aún desconocida. Según Hennon et al. (1990ab, 1994) el declinamiento se presenta en bosques prístinos, puede ocurrir rápidamente (2-3 años) o lentamente en el tiempo (15 años o más) y las áreas afectadas varían en tamaño. Independientemente de cuán rápido se produzca el declinamiento de la copa, el sistema de raíces siempre está afectado al manifestarse los síntomas aéreos. La asociación entre estas dos enfermedades se ha ido acentuando en el tiempo y, en cierta forma, la investigación realizada en $C$. nootkatensis ha servido de base para direccionar los estudios en el "mal del ciprés".

Beever et al. (1996) realizan una interesante revisión sobre la búsqueda de la causa del declinamiento de Cordyline australis (Forster) Endl., detallando sintomatolo- gía, distribución, epidemiología, hipótesis y búsqueda de posibles patógenos. El esquema de trabajo planteado es amplio y complejo, con una gran variedad de técnicas y experimentos utilizados en la investigación. La complejidad en el diseño de actividades deriva de la poca especificidad de los síntomas observados y de la necesidad de evaluar una gran cantidad de hipótesis. La semejanza con el "mal del ciprés" es evidente en este sentido, ya que la sintomatología es muy poco específica y diferentes agentes podrían dar origen a los mismos síntomas.

Otras dos enfermedades que pueden ser relacionadas con el "mal del ciprés" son el declinamiento de Acer saccharum Marsh. y de Pinus taeda L.. La etiología es aún desconocida en ambos casos, aunque en el declinamiento de $P$. taeda se han hecho importantes avances. Hay disponible mucha información sobre ambas enfermedades, entre las que se seleccionaron las revisiones de Minorsky (2003) y Hess et al. (2002). Un punto de especial interés en el declinamiento de $P$. taeda es la aplicación de protocolos y estándares del Forest Health Monitoring (FHM) Program (Hess et al. 2002), y la comparación de la presencia de patógenos en suelo y raíces con indicadores FHM y de crecimiento de árboles. Parámetros como la relación de copa viva, la exposición de la copa a la luz, la densidad, dieback y transparencia de la copa son utilizados. En el declinamiento de $A$. saccharum se evalúa el efecto de insectos defoliadores en la enfermedad (Horsley et al. 2000). Fuertes correlaciones se obtienen entre el número de eventos o el índice de severidad de defoliación y el estado sanitario. Se establece que el efecto de la defoliación en los carbohidratos no estructurales de la planta (especialmente las reservas de almidón de las raíces) juega un rol crítico en el vigor del árbol. La defoliación y la sequía son asociadas con cambios bioquímicos que harían a la planta más susceptible al ataque por organismos secundarios. Importantes estudios son realizados para evaluar la relación entre la defoliación y la nutrición foliar. Algunas de estas evaluaciones o esquemas de trabajo deberían considerarse en el estudio del "mal del ciprés".

Otras enfermedades de especies forestales podrían ser comparadas y relacionadas con el "mal del ciprés" (Wargo et al. 1983, Sherald 1999, Ayres y Lombardero 2000, O’Brien et al. 2002, Roberts 2004). Una evaluación exhaustiva de estas comparaciones no puede ser incluida en este trabajo; sin embargo, sería interesante considerarlas ya que podrían surgir nuevas ideas o diseños experimentales con aplicación al "mal del ciprés".

\section{DISCUSIÓN Y CONCLUSIONES}

El manejo sistemático de la información, logrado al aplicar la guía de diagnóstico, permitió establecer que los datos existentes son aún insuficientes para discriminar entre los diferentes grupos de agentes de daño. Si 
bien es posible identificar una planta afectada, tanto por síntomas externos como internos, falta determinar la relación entre estos síntomas y los problemas a nivel de raíz. El patrón de defoliación que presentan las plantas está asociado a afección de las raíces. Sin embargo, aún no se ha establecido la causa de esta pérdida de hojas. ¿Está el origen de la defoliación en una afección inicial de raíces debida al efecto del anegamiento, a una necrosis crónica producida por hongos pitiáceos o algún otro agente? ¿El sistema radicular se ve afectado por un bloqueo de vasos floemáticos derivado de una infección viral o de bacterias fastidiosas de floema? ¿Cuál es la relación que existe entre la caída en la tasa de crecimiento, la afección de raíces, el amarillamiento y la defoliación? ¿Son todos estos procesos debidos a un mismo agente o diferentes agentes actúan en distintas etapas condicionando el patrón de desarrollo? La pérdida de hojas avanza, por lo general, de la base de la copa hacia arriba y desde el tronco hacia la periferia (Havrylenko et al. 1989, Rajchenberg y Cwielong 1993). ¿Cuáles son los procesos fisiológicos que determinan este patrón? ¿Por qué se produce un avance gradual que involucra inicialmente hojas maduras, reteniéndose en los períodos finales sólo hojas jóvenes? El análisis del patrón de defoliación es un requisito básico para definir la naturaleza del daño. Responder a las preguntas que surgen de la observación del mismo, sería clave para avanzar en el estudio.

Un sistema de asignación de patrones y tipos de vitalidad, común para todos los grupos de investigación, tendría que ser establecido. Dicho sistema no deberá basarse sólo en determinaciones visuales que pueden variar con el observador. La combinación de una serie de parámetros cuantificables en un índice parece una opción más objetiva. Un análisis sobre la utilidad de algunos de estos parámetros puede verse en Bussotti et al. (2002) o en Zarnoch et al. (2004). Es necesario, además, evaluar y determinar la relación que existe entre el patrón de marchitamiento progresivo y el patrón de afección rápida.

La falta de estudios sobre la propagación del disturbio limita la discriminación entre grupos de agentes de daño. La caída en la tasa de crecimiento radial es el síntoma más objetivo del inicio y avance del "mal del ciprés", y debería ser utilizada como base para los estudios de presentación y dispersión de la misma. Alternativamente, un estudio de propagación podría ser realizado en forma comparativa utilizando datos dendrocronológicos y datos de síntomas externos. Estudios complementarios, a través de fotos aéreas, permitirían analizar la propagación y ayudarían a definir si los síntomas internos y externos son o no debidos al mismo agente y cuál es la relación existente entre ellos. Todas estas evaluaciones serán fundamentales en el diseño de estrategias que permitan avanzar en la discriminación del origen biótico, abiótico o mixto del disturbio.

La comparación entre el "mal del ciprés" y otras enfermedades de especies forestales ha permitido compro- bar que son muchas las dificultades que encierra el diagnóstico y el manejo de tales patologías. Se han presentado ejemplos de enfermedades con sintomatología similar que son debidas a diferentes agentes de daño y que han requerido para su identificación la aplicación de técnicas y metodologías diversas. Holdenrieder et al. (2004) presentan una interesante revisión acerca de las enfermedades de especies forestales, haciendo hincapié en los desafíos que plantea la investigación de estas patologías cuando el problema se presenta a escala de bosques. Algunas herramientas cuantitativas y estrategias de investigación que podrían ser de utilidad son comentadas en dicho artículo.

La participación de agentes bióticos (distintos de los hongos) en la mortalidad de A. chilensis no está siendo investigada y debería ser tenida en cuenta. El efecto que la presencia del áfido Cinara tujafilina tiene sobre $A$. chilensis en Argentina y el papel que desempeña en el disturbio no han sido aún evaluados. El análisis de la química foliar y el estudio de los efectos que las condiciones de sitio tienen en la nutrición de la hoja y a su vez en los sistemas de defensa de la planta tampoco han sido desarrollados para el "mal del ciprés".

Los enfoques planteados en el trabajo han permitido identificar algunos puntos clave que deberían ser considerados en investigaciones futuras, entre los que se encuentran: falta de especificidad de síntomas y de estudios de propagación, ausencia de signos, subjetividad en la asignación del grado de defoliación y el estado sanitario asociado, falta de un modelo de análisis para el disturbio.

Es necesario, en síntesis, continuar la investigación del "mal del ciprés" teniendo en mente la complejidad del estudio de este tipo de disturbios forestales. La falta de signos, la falta de especificidad de los síntomas y la dificultad para establecer la asociación espacial y los patrones de dispersión o propagación de la afección obligan a considerar la participación de diferentes agentes de daño (bióticos, abióticos o mezcla de ambos) en el desarrollo del proceso y a diseñar ensayos o experimentos que sean lo suficientemente robustos como para responder a los complejos interrogantes planteados.

Es importante señalar que a la luz de la investigación realizada hasta la fecha la denominación "mal del ciprés" parece ser la más adecuada para el disturbio que afecta a la especie A. chilensis en Argentina. Esta denominación hace referencia a un síndrome complejo desde el cual, probablemente, sea posible discriminar diferentes disturbios. Cada uno de éstos, una vez definido, deberá ser nombrado de acuerdo con su agente causal.

\section{AGRADECIMIENTOS}

A la Dra. Alina Greslebin (CIEFAP, Centro de Investigación y Extensión Forestal Andino Patagónico) por el apoyo recibido y el constante intercambio de ideas. Al 
Dr. Leonardo Gallo (INTA, Instituto Nacional de Tecnología Agropecuaria) y al Dr. Mario Rajchenberg (CIEFAP) por la lectura y las recomendaciones sobre el manuscrito. A todos aquellos que anónimamente colaboraron con nuestro trabajo. La financiación del presente artículo corresponde al Proyecto Nacional 520405: Funciones y algoritmos dasométricos para manejo silvícola intenso, de aplicación en plantaciones forestales orientadas a producción de madera de alto valor agregado.

\section{REFERENCIAS}

Ayres M, M Lombardero. 2000. Assessing the consequences of global change for forest disturbance for herbivores and pathogens. Sci Total Environ. 262: 263-286.

Baccalá NB, PH Rosso, M Havrylenko. 1998. Austrocedrus chilensis mortality in the Nahuel Huapi National Park (Argentina). Forest Ecol. Manag. 109: 261-269.

Beever R, R Forster, J Rees-George, G Robertson, G Wood, C Winks. 1996. Sudden decline of cabbage tree (Cordyline australis): Search for the cause. New Zealand Journal of Ecology 20(1): 53-68.

Bussotti F, A Cozzi, M Ferretti, E Cenni, D Bettini, R Nibbi. 2002. Crown condition assessment at the CONECOFOR Permanent Monitoring Plots. Long-term ecological research in Italian forest ecosystems. J. Limnol. 61(1): 12-18.

Calí SG. 1996. Austrocedrus chilensis: Estudio de los anillos de crecimiento y su relación con la dinámica del "Mal del ciprés" en el Parque Nacional Nahuel Huapi, Argentina. Tesis Licenciado en Biología. Bariloche, Argentina. Universidad Nacional del Comahue. 143 p.

Dezzotti A, L Sancholuz. 1991. Los bosques de Austrocedrus chilensis en Argentina: ubicación, estructura y crecimiento. Bosque 12: 43-52.

Filip GM, PH Rosso. 1999. Cypress mortality (mal del ciprés) in the Patagonian Andes: comparisons with similar forest diseases and declines in North America. Eur. J. For. Path. 29: 89-96.

Gallo L, MJ Pastorino, C Donoso. 2004. Variación en Austrocedrus chilensis (D. Don) Pic. Ser et Bizzarri (Ciprés de la Cordillera). In Donoso C, R Ipinza, A Premoli, L Ga1lo. Variación intraespecífica en las especies arbóreas de los bosques templados de Chile y Argentina. Chile. Editorial Universitaria. p. 233-252.

Green J, O Maloy, J Capizzi. 2003. A systematic approach to diagnosing plant damage. Consultado marzo 2005. Disponible en http://oregonstate.edu/Dept/nurspest/ otherresources.htm

Greslebin AG, EM Hansen, LM Winton, M Rajchenberg. 2005. Phytophthora species from declining Austrocedrus chilensis forests in Patagonia, Argentina. Mycologia 97(1): 218-228.

Havrylenko M, PH Rosso, SB Fontenla. 1989. Austrocedrus chilensis: contribución al estudio de su mortalidad en Argentina. Bosque 10(1): 29-36.

Hennon P, M Rajchenberg. 2000. El "mal del ciprés". Algunas observaciones, comparaciones e ideas. CIEFAP-Patagonia Forestal 2: 4-6.
Hennon P, E Hansen, C Shaw III. 1990a. Dynamics of decline and mortality in Chamaecyparis nootkatensis in southeast Alaska. Can. J. Bot. 68: 651-662.

Hennon P, C Shaw III, E Hansen. 1990b. Dating decline and mortality of Chamaecyparis nootkatensis in southeast Alaska. Forest Science 36: 502-515.

Hennon P, M Schultz, D Wittwer. 1994. Yellow-cedar decline. Consultado marzo 2005. Disponible en http://www.fs.fed.us/ r10/spf/fhp/sefhp/seycedar.htm

Hess N, W Otrosina, E Carter, J Steinman, J Jones, L Eckhardt, A Weber, C Walkinshaw. 2002. Assessment of loblolly pine decline in central Alabama. In Proceedings of the eleventh biennial southern silvicultural research conference. Gen. Tech. Rep. SRS-48. Asheville, NC: U.S. Department of Agriculture, Forest Service, Southern Research Station. p. 558-564.

Holdenrieder O, M Pautasso, P Weisberg, D Lonsdale. 2004. Tree diseases and landscape processes: the challenge of landscape pathology. Trends Ecol. and Evol. 19: 446-452.

Horsley S, R Long, S Bailey, R Hallett, T Hall. 2000. Factors associated with the decline disease of sugar maple on the Allegheny Plateau. Can. J. For. Res. 30: 1365-1378.

Hranilovich S. 1988. Informe Histórico sobre el "mal del ciprés" de la cordillera (Austrocedrus chilensis). Revista de la Asociación Forestal Argentina. Año XLII. 3: 58-62.

La Manna L, M Rajchenberg. 2004a. The decline of Austrocedrus chilensis forests in Patagonia, Argentina: soil features as predisposing factors. Forest Ecol. Manag. 190: 345-357.

La Manna L, M Rajchenberg. 2004b. Soil properties and Austrocedrus chilensis forests decline in Central Patagonia, Argentina. Plant and Soil 263: 29-41.

La Manna L, M Rajchenberg, L Taladiz. 2001. El "Mal del ciprés" en el Valle 16 de Octubre: ¿los suelos pueden decirnos algo? CIEFAP-Patagonia Forestal. 7: 11-12.

Minorsky P. 2003. The hot and the classic. The decline of sugar maples (Acer saccharum). Plant Physiol. 133: 441-442.

O’Brien J, M Mielke, S Oak, B Moltzan. 2002. Sudden oak death. Pest alert. United States Department of Agriculture, Forest Service. NA-PR-02-02. Consultado marzo 2005. Disponible en http://www.na.fs.fed.us/spfo/pubs/pest_al/ sodeast/sodeast.htm

Rajchenberg M, PP Cwielong. 1993. El mal del ciprés (Austrocedrus chilensis): su relación con las pudriciones radiculares y el sitio. CIEFAP, Anales Volumen 1. p. 96-108.

Roberts D. 2004. Ash tree decline. Michigan State University Extension. Consultado marzo 2005. Disponible en http:// web1.msue.msu.edu/reg_se/roberts/ash/decline.html\#what

Rosso PH, N Baccalá, M Havrylenko, S Fontenla. 1994. Spatial pattern of Austrocedrus chilensis wilting and the scope of autocorrelation analysis in natural forests. Forest Ecol. Manag. 67: 273-279.

Rosso PH, M Havrylenko, S Fontenla. 1989. Austrocedrus chilensis: asociación espacial entre individuos sanos y afectados por la mortalidad. Bosque 10(2): 85-88.

Sherald J. 1999. Elm yellows. Park Science 19 (2). Consultado marzo 2005. Disponible en http://www2.nature.nps.gov/ parksci/vol19/vol19(2)/10sherald.htm 
Varsavsky E, L Bettuci, D Rodríguez García, C Gómez. 1975. Observaciones preliminares sobre la mortalidad del ciprés (Austrocedrus chilensis) en los bosques patagónicos. Fundación Bariloche, publicación $\mathrm{N}^{\circ} 19.11 \mathrm{p}$.

Wargo P, D Houston, L LaMadeleine. 1983. Oak decline. Forest insect and disease leaflet 165. Department of Agri- culture, Forest Service. Consultado marzo 2005. Disponible en http://www.na.fs.fed.us/spfo/pubs/fidls/ oakdecline/oakdecline.htm

Zarnoch S, W Bechtold, K Stoke. 2004. Using crown condition variables as indicators of forest health. Can. J. For. Res. 34: $1057-1070$.

Recibido: 26.07.05

Aceptado: 12.01 .07 